\title{
The Psychosocial Factors that Influencing HIV-AIDS Antiretroviral Treatment Adherence
}

\author{
Liranso $\mathrm{GS}^{*}$, Mohan $\mathrm{SS}^{2}$ and Eyerus $\mathrm{A}^{3}$
}

${ }^{1} \mathrm{PhD}$ Scholar in Social Work, Mangalore University, India

${ }^{2}$ Asst. Professor, Research guide, \& Journal editor in Social Work, Mangalore University, India

${ }^{3}$ Social Work researcher and ART practitioner in Alert Hospital, Addis Ababa, Ethiopia

*Corresponding author: Liranso GS, PhD Scholar in Social Work, Mangalore, India, Email: liranso.2006@ gmail.com

Citation: Liranso GS, Mohan SS, Eyerus A (2016) The Psychosocial Factors that Influencing HIV-AIDS Antiretroviral Treatment Adherence. J Aids Hiv Infec 2(2): 205. doi: 10.15744/2454-499X.2.205

Received Date: October 01, 2016 Accepted Date: December 15, 2016 Published Date: December 19, 2016

\begin{abstract}
The socio-cultural context of illness has become a major research area, and it has made significant contributions to our understanding of the socio-cultural dimensions of illness. In this article, we briefly trace the roots of a socio-cultural approach to illness, and we present some of the key findings of socio-cultural organized under main themes. Adherence to antiretroviral therapy drugs in the treatment of HIV infection is complicated because of many psychosocial factors. The aim of the study was to investigate the psychosocial factors involved in non-compliance with ART among people infected with HIV in Alert Hospital in Ethiopia. To achieve its objective, the study utilized a qualitative method to gather direct, inductive and wealthy information from the participants. The study covered interviews with twenty participants. The participants were selected using purposive sampling technique. The collected data was categorized and analyzed thematically based on the research objective. The results of the study indicated that the major psychological and social factors such as stigma and discrimination, social support, substance abuse, subjective norms, belief system and perceived behavioral control, and various cultural factors may uniquely contribute to deeper and potential barriers to ART adherence. Evaluating changes in the level of knowledge health workers trained in adherence measurements and concerned bodies work for providing relevant and updated information about ART issues for the followers in a convincing way. Therefore, the study has concluded that health workers in all facilities that provide ART would be trained in adherence counseling through formal training.
\end{abstract}

Keywords: Antiretroviral Therapy; Treatment; Adherence and Psychosocial Factors

\section{Introduction}

The Human Immune Virus (HIV) has created a vast challenge throughout the world. A report at end of 2012 showed that approximately 34 million people were living with HIV worldwide [1]. The epidemic continues to be more severe in Africa than any other country in the world. Almost half of the deaths from Acquired immune deficiency syndrome (AIDS) related illness in 2012 occurred in Africa. According to HAPCO, 1.1million people are living with HIV with Marked regional variations [2]. AIDS is one of the most destructive epidemics the world has ever witnessed. Presently an estimated 33.4 million people are living with HIV worldwide, nearly two-thirds of these live in sub-Saharan Africa. Antiretroviral therapy (ART) has shown to delay progression to AIDS, resulting in a greater and more sustained virologic and immunologic response and improve survival [1].

In sub-Saharan Africa, there has been a dramatic increase in the number of HIV/AIDS patients on antiretroviral treatment from just 100,000 persons in 2003 to 3.9 million in 2009 involving close to $40 \%$ of those in need of the treatment. Two sub-Saharan Africa countries, Botswana and Rwanda, have achieved universal access target (treatment coverage of $80 \%$ or more of patients in need) at the end of 2009, while countries such as Ethiopia, Zambia, Namibia, and Senegal are moving closer to the same target having covered $50-80 \%$ of patients in need of treatment [3]. Since access to ART is vital, it is equally important to ensure the patients adherence to the prescribed regimen. Adherence in this sense is defined as the "extent to which client's behavior matches with the prescribed health care regimen as agreed through a shared decision making process between the client and the health care provider" [4]. Successful ART provision thus requires not just medical attention alone but also long term social and psychological support, including encouraging and monitoring adherence [5].

Appropriate use of antiretroviral therapy (ART) has improved the health of many HIV positive individuals otherwise may have died. Notably, the efficacy of any treatment depends on sustained high levels of adherence to ART. But ART regimens are often 
complicated and can include varying dosing schedules, dietary requirements, and adverse effects. Adherence is a major predictor of the survival of individuals living with HIV/AIDS and poor adherence to treatment remains a major obstacle in the fight against HIV. From the perspective of service delivery, treatment non-adherence undermines the efficient distribution of resources and huge expenditure by international organizations including the United States President's Emergency Plan for AIDS Relief (PEPFAR), the Global Fund and the World Bank [6].

According to WHO, the interventions target adherences was tailored to the demands that experienced by the patients with that specific illness [7]. As a result, it is important for health care providers to assess those factors that impact negatively on adherence. In order to improve adherence the health care providers should first ascertain treatment readiness, otherwise treatment may be given to patient who are not ready to follow the regime. In addition, patients should be advised on how to take their treatment. Patients who show less than $80 \%$ compliance require increases adherence support knowledge regarding the effect of Anti retroviral medication on viral load is a better predictor of adherence than the patient's level of education [8].

There are several psychosocial factors that are directly or indirectly affecting adherence. These are social and psychological factors may lead to non adherence has serious consequences both on the individual and the society. If there is poor adherence then there is a higher risk that drug resistance will develop, which will result in need for second-line drug treatment. When people living with HIV require second line treatment, it can be ten times more expensive than first line drugs [7].

Studies have however shown that youth are more prone to poor adherence increasing their risk and heightening risk for early mortality. Globally, young people constitute more than half of those infected by HIV with reports estimating that over 6000 youth get infected with HIV every day [9]. This increasing number of young people getting infected means there are more young people being initiated into treatment programs. Behaviours associated with adherence such as taking doses at the same time every day, following food restrictions and skipping doses as a result of irregularity in routines remain a challenge to youth living with HIV [10].

The socio-cultural context of illness has become a major research area, and it has made significant contributions to our understanding of the socio-cultural dimensions of illness. In this article we briefly trace the roots of a socio-cultural approach to illness, and we present some of the key findings of socio-cultural organized under main themes. HIV medications can have some significant, long-term side effects. While many of these side effects are treatable, some can be long-term. The client needs to tell the healthcare provider about any side effects, so that he or she can decide the best course of treatment for both the HIV disease and the side effects. Most of those involved in the movement to slow the spread of AIDS and to improve the quality of life of those living with HIV view stigma and discrimination as human rights violations requiring redress. Both organizations and individuals have taken various actions to address stigma; however, these actions often have not been grounded in a broad biosocial understanding of stigma and AIDS-related discrimination [11].

In Ethiopia primary health care Kagee resulting in high rate of relapse rehospitalisation of disease and mortality [12]. Nonadherence studies are still in the exploratory phase, especially in Ethiopia and as a result, explanations for this phenomenon are not yet fully developed. Adherence that is sub-optimal results in replication of the virus, which promotes the development of drug resistance HIV-variants, leading to treatment failure as well as limited options for future therapy [13]. Therefore, this study was tried to assess the psychosocial factors affecting ART adherence among young people living with HIV in Alert Hospital in Ethiopia.

The result of the study may also help those Hospitals and organizations working on more effectively and efficiently service empower, and use internal and external resources to develop strong sustainable programmatic responses to minimize the psychosocial factors that influence ART adherence among PLWHA in sustainable ways. Besides, it is hoped that the results of this study may provide some information on the present psychosocial factors that influence ART adherence for those who are interested in conducting further investigation on the issue under discussion. Moreover, the study may help to identify the strengths and weaknesses regarding ART service challenges. This will be built upon the strengths and work hard to improve the shortcomings.

Finally, since the relevant and feasible recommendations are forwarded to add scientific values on the existing strategies, programs, services and practices specific to the present psychosocial factors that influence ART adherence which will help to enhance the psychosocial wellbeing of ART stakeholders, all concerned government agencies, NGOs, the academic community, and associations working on ART and ART participants themselves benefit from this study.

\section{Methods}

The research design refers to the structure of an inquiry whose role is to minimize the chance of drawing incorrect causal inferences from the data [14]. The study was employing a qualitative method to make the description of the existing psychological and social factor that affects ART Adherence in Alert hospital. There are a number of reasons for choosing a qualitative research methodology for this particular study. Firstly, qualitative research is considered to be an appropriate methodology for researchers whose research questions lead them towards an 'inductive' or 'data-driven' approach- that is, to look at what is going on and try to make sense of that by testing out themes and patterns. Secondly, the qualitative method of data collection and analysis provides rich information and meanings which are not always accessible through the quantitative method of data collection [14]. 
In this study, two data sources were employed, namely, young people between 25-35 years-old twenty participants were included in the study to get more effective responses to the psychosocial factors involved in not- compliance with ART among people infected with HIV in the Hospital. Because it was assumed that their understanding level was good to describe their experiences. Moreover, young people undergo a developmental period of experimentation, risk taking and confronting a host of choices with regard to identity formation, relationships, sexual behaviour and drugs and substance abuse [10].

According to Durrhin \& Painter, purposive sampling is often used when looking for rare and particular types of participants [15]. In light of this, the sampling technique was employed to identify all of the respondents with rich information appropriate for this study. Young people living with HIV, 25-35 years-old twenty participants were selected using a purposive sampling technique. Through these associates and references of the participants, it was finally possible to accumulate a sufficient data to check more deeply on the psycho social factors involved in non-compliance with ART among people infected with HIV in the study area.

To collect data from the respondents, the researchers utilized semi-structured interview. According to Mugenda (2003), semistructured interview is easier to answer and analyze for unambiguous responses [16]. Furthermore, a great advantage of the semi-structured interview, according to Kruger as cited in Yolandi mentioned that is its flexibility, which allows the researchers to grasp more fully the participant's practice than would be possible through the implementation of a more rigid methodological technique $[17,18]$. Based on this, a semi-structured interview guide was drawn up for each interview in order to elicit and acquire rich information relevant to meet the objective of the study.

According to Durrhin and Painter, data collection procedures refer to the process followed by the researcher to collect the necessary information [15]. The researcher with the help of coordinator of the Hospital contacted and selected participants'. Selected participants were informed and asked their permission to participate in the study.

To obtain an informed consent from the respondents, the researchers explained the consent form which includes issues about the purpose of the research, confidentiality, rights of informants, duration of discussions, and note taking. Hence, most of the respondents agreed to participate in the study. The average amount of time the interviews took with each participant was from 45 to 55 minutes.

The interviews were conducted in collaboration with one staff member who has a strong attachment with the researchers. After the respondents were selected they were interview by using the Amharic version of the interview guided by the researchers. After the data was collected, it was transcribed from Amharic to English. The dictations and translations were also carefully reviewed for consistency. Some corrections were made. Then transliterates were read numerous times to gain better understandings of the responses of participants. Field's notes were translated from Amharic to the English language. The transcribed data was organized in themes according to the research questions themes. Then after, the findings of the study were analyzed based on the psychosocial factors involved in not-compliance with ART among people infected with HIV.

Moreover, a themes and sub-themes were discussed in categorized and summarized forms of major themes based on the research questions of the study. Finally, the research result categories or clusters were summarized into major themes. Some selected respondents' stories were presented in quotations to see the turning points of psychosocial care and support issues. Hence, thematic data analysis was employed in this study. As (Kumar, 2008), stated theme analysis was a good fit for qualitative research [19].

\section{Results}

The purpose of the study was to explore influence of psychosocial factors on ART adherence among young people living with HIV in Alert Hospital. This part of study presents the major findings as follows.

\begin{tabular}{|c|c|c|c|c|}
\hline \multirow{3}{*}{$\begin{array}{c}\text { Level of } \\
\text { Education }\end{array}$} & \multicolumn{4}{|c|}{ Sex } \\
\hline & \multicolumn{2}{|c|}{ Male } & \multicolumn{2}{|c|}{ Female } \\
\hline & No & $\%$ & No & $\%$ \\
\hline Illiterate & 2 & 10 & 3 & 15 \\
\hline Grade 1-4 & - & - & 2 & 10 \\
\hline Grade 5-8 & 1 & 5 & 2 & 10 \\
\hline Grade 9-12 & 3 & 15 & 3 & 15 \\
\hline Grade $12+$ & 2 & 10 & 2 & 10 \\
\hline Total & 8 & 100 & 12 & 100 \\
\hline
\end{tabular}

Table 1: An overview of sex and educational background information of respondents

In table 1: an overview of sex and educational background information on respondents. Among the total twenty participants, five of them (two male and three females) were illiterate. Only two female participants have an elementary education. In addition, four (one male and two females) of the participants are educated at junior high school level (grade 5-8). Furthermore, eight (three males and three females) have attended high school education (grade 9-12) while four (two males and two females) have attended Grade 12+ levels. Table 2: an overview of religion and marital status information of the participants described. 


\begin{tabular}{|c|c|c|c|}
\hline \multicolumn{2}{|c|}{ Dimensions } & No & $\%$ \\
\hline \multirow{4}{*}{ Religion } & Christian & 12 & 60 \\
\cline { 2 - 4 } & Muslim & 6 & 30 \\
\cline { 2 - 4 } & Other & 2 & 10 \\
\cline { 2 - 4 } & Total & $\mathbf{2 0}$ & $\mathbf{1 0 0}$ \\
\hline \multirow{4}{*}{$\begin{array}{c}\text { Marital } \\
\text { Status }\end{array}$} & Single & 8 & 40 \\
\cline { 2 - 4 } & Married & 6 & 30 \\
\cline { 2 - 4 } & Divorced & 4 & 20 \\
\cline { 2 - 4 } & Widowed & 2 & 10 \\
\cline { 2 - 4 } & Total & $\mathbf{2 0}$ & $\mathbf{1 0 0}$ \\
\hline
\end{tabular}

Table 2: An overview of religion and marital status information of the participants

Regarding the data collected on the religious background of the participants show that twelve were Christian, six were Muslims and while two was other. Furthermore, the data procured on backgrounds related to the marital status of the respondents show that eight of the participants were single, six were married, four were divorced, and other two of the participants were windowed.

\section{The Psychosocial Factors that Influence ART Customers' Adherence}

Almost all participants reported that feeling of depression; anger and suicide are presented because of their tendency to occur either in isolation in reaction to being diagnosis with HIV. After being diagnosis with HIV, the participant's reaction to the news varied from depression, anger, denial, fear and suicide to acceptance; most of the participant who experienced difficulty. They did not get counselling, adhered poorly to the medication on refused to take ART.

Also, six participants have reported that their spouse refuse to use condoms. The initial reaction of most of the women to the diagnosis was acceptance; the difficulty was with their partners. They refused to get tested for HIV and insisted that they were not sick, so they continue living as if nothing has changed. In line with this:

\section{I lost my boyfriend before two years by AIDS-related illness. But, I was diagnosed while I was pregnant $(F m, 2)$.}

Furthermore, fear of dying and not being able to raise their children was expressed by many of the participants. Three participants were mentioned that they had been through stages where they experienced fear constantly. They attributed this to a fear of rejection and dying. Thoughts of leaving their children without a mother, being unable to raise them, and leaving them without someone who would love and take care of them it is the source of many women fear.

I refuse to take ART medication, because, I believe that it exposed me to suffer anxiety and depression. Also, I don't accept the conditions. Already, I was affected psychologically $(F m, 8)$.

The information collected from participants also shows that they had not accepted that they were HIV positive by the time that they were interviewed. Because they stated that they already accepted HIV as part of their lives. Also, the data shows that adherence behaviour such as regular follow- ups and taking the medication as prescribed indicated a degree of acceptance of the diagnosis.

The following extract, from one male participant, illustrates the more:

Already, I know my status before one year back, I live alone, most of the time I easily become hopeless and suffer depression because of disease condition and isolation. Also, I lose my weight and exposed to body weakness $(M p, 6)$.

Other participants also indicated that abused by sexually, physically and because of refuses to use condoms. In line with this, the following quote, illustrates the above challenges of the participants:

Currently, I live with my sister. My husband passed away by AIDS-related illness before five years ago and then I was diagnosed. I believed that I have infected because of a refusal of condoms, I also abused physically $(F P, 5)$.

However, the following citation illustrates inconsistent with the above:

I see ART as right, but you know, everyone goes with the belief of a person if a person believes that it will help, but if a person is not trusting it, has a negative belief about it, then it will not help (FP, 7).

Furthermore, respondents illustrated that they were experienced different psychological problems such as stigma and rejection from the society. The participants mentioned the fear of being stigmatized and others finding out that they were HIV-positive 
as reasons for their social withdrawal as well as for their unwillingness to collect their medication. They were reluctant to take their medication because they fear being seen there by people who know them. As a result, these participants prefer to keep to themselves alone. The following citation strengthens this idea:

"I disclose my real status, especially to those who don't know me. Because I believed that they talk about me in their groups and when I join them they act as if they don't mind" $(M p, 1)$.

Moreover, the following citation also illustrates more in relation to the above:

"I cannot take my drugs when people are seeing. I always go and hide and take them. Otherwise, people start to speak very softly about me all the time (FP, 3).

The other important thing that emerged from the participants was the refusal of condom usage. Most of the participants stated that they do not want to use condoms because it is against their culture. They also reported that using condoms removes the joy of sexual experience; all of these participants stated that they never used condoms before being diagnosed. Additionally, all the participants indicated that experiencing side effects from the medication. This was also one of the reasons given for noncompliance.

The unpleasant nature of the side effects, especially sickness, vomiting, weakness, diarrhea these side effect increase reluctance to adhere well.

\section{"My mother has enough knowledge about traditional medicine. She influenced me to use traditional medicine and she was told to discontinue antiretroviral medication while taking the traditional medication" (FP, 4).}

According to Ayalu \& A. Reda, currently, there is no gold standard for measuring adherence [3]. Because of this, most of the studies included used the most common forms of adherence assessment patient recall and pill count which have recognized ART medication. It is further challenged by various social and clinical obstacles where inadequate suppression of viral replication by ART are resulting due to poor adherence to therapy, the low potency of the antiretroviral regimens, viral resistance to antiretroviral medications, and pharmacokinetic interactions causing inadequate drug delivery.

Moreover, there are only very few studies that investigate adherence. The aim of the research was to assess the challenges of adherence to ART and to identify the factors that contribute to poor adherence. Current estimates of adherence studies indicate that despite earlier fears of poor medication adherence.

In line with this, the following quote illustrates the above challenge of ART medication.

Many people told me many things. They told me that the medicine has very shocking behaviour; it claims life while on sleep; it causes blindness and deafness; it results in bruises and it removes hair; and so on. Therefore, for the fear of such bad things I kept the medicine with me without taking it for many days $(M p, 7)$

Furthermore, the above-aforementioned reality in line with Ayalu \& A. Reda, research result that health system barriers affect adherence, especially a regular and timely supply of medication to patients [3]. An unreliable supply of medications can severely reduce patient adherence rates. These include not use of new monitoring mechanisms, infrastructure, staffing, training of counsellors, community support systems, and suitable drug formulations.

All respondents reported disclosure of their status to others. In fact, except one participant reported that they had someone in their household who knew about their treatment. Respondents rarely reported stopping the medication because of family pressure or stigma.

\section{Liranso Adherence Counselling Model}

Although one subject indicated that fear of stigma in the workplace contributed to his inability to request time off to collect medication, another respondent stated that pressure and fear of disclosure did not affect her decision:

One of the reasons I stopped treatment might be because of transportation costs, but not side effects or fear of disclosure, because there are people in my street who know my status and my friends. No, no one has pressurized me to stop my pills (FP, 3).

In contrast to other studies in the literature, the cost of medication was not an important barrier among respondents because patients obtained ART and healthcare at clinics free of charge. However, transfer patients reported that transportation costs influenced treatment retention. The majority of transfer patients lowered transportation costs by changing clinic. Possible interventions to overcome financial barriers include providing stipends or vouchers to patients to cover transport costs. Additionally, a treatment 
plan designed to accommodate less frequent appointments and drug pick up, could reduce patient costs and reduce the time burden that ART places upon patients.

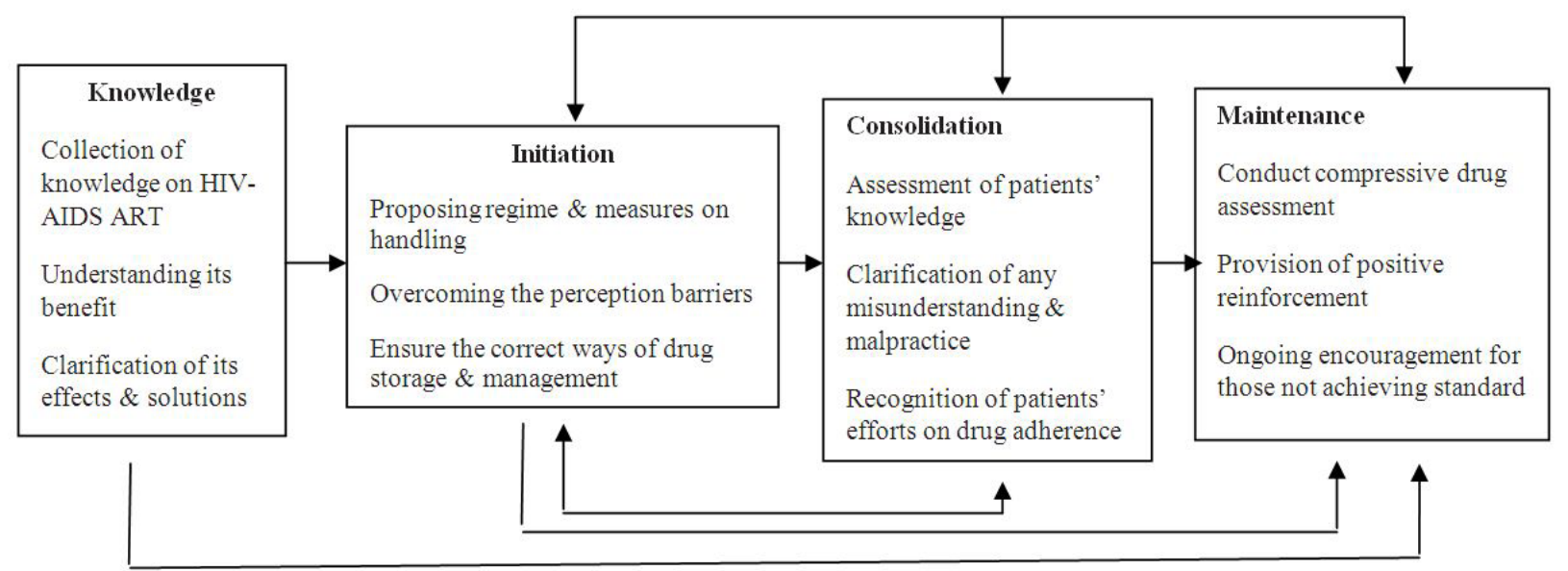

Figure 1: Liranso Model of Adherence counselling modified from HAART

On the other hand, most participants informed that rejection and stigmatization after disclosing the condition. They lost loved one because of HIV, rejection, and stigmatization from other has been described as contributing to non-compliance. In addition, they illustrated that negative reactions from community members make them withdraw entirely from any contact with the community, and rely entirely on the support of family members, as one respondent confided:

\begin{abstract}
At first, I got so scared to tell people because of the stigma but ever since I started on ART they have begun to wonder what has happened. In fact, I hear some say why they don't inject her and put her to rest otherwise she is going to finish everybody. Because of these, I remained alone. So I remained with only a few of my family members who I cooperate with. My intimate friend advised me to tell our family members about it. In fact, these helped me so much when I told them I became free about my status and up to now I have a lot of courage and strength with the ART (FP, 8).
\end{abstract}

Furthermore, the following citation also illustrates more in relation to the above:

"My wife abandoned me the moment I disclosed my status to her. She left me with the children. And to make it worse, my own family said I deserved harsh treatment because I infected my wife with the fatal virus." (Mp, 3)

Additionally, they indicated that the involvement of family, friends and others in helping the patient to deal with the disease. In some cases the family members distanced themselves from issues pertaining to HIV/AIDS, better understanding from family and friends has been cited as being helpful in improving compliance to treatment.
"My children after seeing the state I was in and after getting ART, I called them and told them about my state. They got encouraged and as a result, they buy me passion fruits and sugar because they know the drugs I am taking are so strong. I even wrote my file number on the wall and told them that just in case I am badly off they can go to the wall and get me help. My children know very well that my drug needs to drink enough and to eat on time. One thing that motivated me to tell them is because I thought I could be so weak to collect my refill of the drug" (FP, 1).

However, not all children are equally supportive, as shown in the following complaint:
"It is only my young children who give help. They cook for me and bring me whatever I send them. But my elder children neglected me yet I told them about my status (FP, 1).

Most participants indicated that the different belief system of participants and their families also emerged as one of the issues that contribute to non-compliance. Lack of trust in the ART medications and trust in spiritual bases were considered. Most of the participant default treatment because of traditional beliefs and become sick as a result. The following excerpt, from one participant, illustrates further:

When I was discussing with one fellow member about ART, she has told me that she lack trust in the ART medications. So, Satan is using the ART to attract the PLWHA into committing faithlessness (Mp, 3). 
Respondents overwhelmingly reported that ART has low improved their health status and quality of life. Nevertheless, despite improved health from taking ART and worse health when treatment is stopped, serious barriers to treatment remained: transport costs, time needed for treatment and logistical challenges were barriers to treatment, whereas stigma around HIV/AIDS and side effects associated with ART were discussed. Also, the participants described that a number of causes that forced them to delay taking the ART on time, because of failure in meeting the needs triggered in relation to food and other prescribed orders of the physician. Some of the major causes stated by participants based on the frequency of response from higher to lower include: involvement in long lasting social responsibility like mourning and wedding; lack of financial support which can be used to cover the food and related needs that have to be responded in the process of taking ART as a lifelong therapy; forgetfulness and long travel were among the causes discussed by participants of the study.

Additionally, participants of the study indicated that because of fear of stigma and discriminations forwarded from their community, they delay the time for taking ART in occasions where the member of the family are around their home for any social reason. In addition, eight participants who said that the nature of their work is one of the major causes that adversely contributed to the delay in taking the medicine on time. They said that because of the attached stigma and discrimination, they do not take their medicine at their workplace for not being seen taking the medicine. Consequently, however, their disclosure has resulted in stigma and discrimination on them.

Therefore, except the PLWHA who haven't disclosed their zero-positive either for their relatives or for their friends, all of the respondents went through a trial of stigma. Besides, respondents tremendously reported that lack of knowledge regarding adherence to the ART regimen also seemed to affect the notion of what to do if one forgot to take ART at the prescribed time, which is essential information to provide when dispensing ART. The majority of the participants were found not to have experienced side-effects during their treatment. Knowledge regarding side-effects seemed not to have been sufficiently covered, with most of the participants still ignoring the possibility that they might experience side-effects, and not concerning themselves about ensuring that they knew what to do if they did experience them.

Furthermore, participants reported that because of the tiresome nature of work they engage in, they sleep earlier and sometimes miss taking the medicine. Few of the participants have stated that the nature of their work in making them tired so that they do not have any time to involve in community affairs.

In addition, as we have seen so far in the findings, the respondents of this study have different knowledge and understandings about HIV/AIDS related issues including ART. It is obvious that their attitude, understanding, and knowledge about the pandemic would have its own impact on the implementation of ART medication.

\section{Discussions}

The study was tried to assess the psychosocial factors affecting ART adherence among young people living with HIV in Alert Hospital. Compliance remains one of the major challenges in managing medication for those patients living with HIV/AIDS. A qualitative research method was applied in order to assess the major psychosocial factors that affect adherence to antiretroviral treatment. The participants were selected using purposive sampling technique. Twenty participants between 25-35 years old were included in the study. The data were collected by means of semi-structured interview and focus group discussion. The data were analyzed thematically. The themes emerged and compared with integrated with the literature. To this end, the study has used indepth interview, and focus group discussion of the researcher to collect data from twenty people who are purposely selected for the study.

Ayalu \& A. Reda also stated that the challenges of adherence to ART identified include factors related to patients and their families, socioeconomic factors, medication, and health care systems were indicated [3]. This has implications for interventions to improve ART adherence and therefore the program needs to address these interrelated and multidimensional factors. In other words, ensuring adherence to treatment and retention requires an understanding of the multiple barriers that patients face and developing interventions that overcome these barriers.

There is consistency between this study and USAIDS stigma and discrimination were among the biggest challenges for HIV patients as they would be reluctant to get treatment, care, and support for fear of alienation [20]. Preventing and reducing stigma is vital so that people are not discouraged from using and helping others on ART. Because of stigma patients were forced to take their medications in secrecy increasing the likelihood of non-adherence.

Likewise, traditional medication is appearing to interfere with ART for most patients although several patients reported taking traditional medication both in addition to and as a substitute for ART. Many patients reported feelings of stress, but few associated their stress with a reduced capacity to adhere to their medication regimen. Some patients reported that negative side effects led them to discontinue treatment. The result has revealed some of the major psychosocial factors that affect adherence to antiretroviral therapy based on self-report of participants. 


\section{Conclusion}

The strength of this study depends on the qualitative data, in which researchers' present views about adherence by reporting statements from the study participants themselves. As we have seen in the findings and discussion parts, HIV/AIDS-related psychosocial factors involved in not-compliance with ART have several implications on the life of the respondents. Particularly in connection with using ART, the respondents have different experiences. Major psychosocial factors involved in not-compliance in using ART are identified and discussed. The results of the study showed that the major psychosocial factors that affect adherence to antiretroviral therapy based on self-report of participants.

Some of the factors are social stigma and discrimination, belief system, depression, anxiety, distress side effects from the medication (lack of trust in the ART medications) were described more. These concerns require consideration in cycle with the patient focused psychosocial concerns raised here. Efforts to minimize constraints and improve adherence levels will require the efforts of the community, health workers, and patients as well as Government commitment to resolve key structural problems.

From the findings, it is also possible to conclude that the psychosocial factors affecting ART adherence among young people living with HIV in Alert Hospital are health illiteracy, stigma, poor social support, substance abuse, attitudes, subjective norms, and perceived behavioural control, and various cultural factors may uniquely contribute to a deeper potential barriers to ART adherence.

\section{References}

1. UNAID (2012) World AIDS Day Report. Geneva, Switzerland.

2. HAPCO (2012) Effect of nutritional factors on adherence to antiretroviral therapy among HIV-infected adults: a case control study in Northern Ethiopia. BMC Infect Dis 13: 233

3. Reda AA, Biadgilign S (2011) Determinants of Adherence to Antiretroviral Therapy among HIV Infected Patients in Africa. AIDS Res Treat doi: $10.1155 / 2012 / 574656$.

4. Steele RG, Grauer D (2003) Adherence to antiretroviral therapy for paediatric HIV infection: review of the literature and recommendations for research. Clin Child Fam Psychol Rev 6:17-30.

5. Hope R. and Israel E., (2007). The essentials of antiretroviral therapy for health care and program managers Pathfinder International, Technical Guidance Series Number 5. Watertown MA: Pathfinder International.

6. Pennington J (2007) HIV/AIDS in Nigeria.

7. Sasaki Y, Kakimoto K, Dube C, Sikazwe I, Moyo C, et al. (2010) Adherence to antiretroviral therapy (ART) during the early months of treatment in rural Zambia: influence of demographic characteristics and social surroundings of patients. Ann Clin Microbiol Antimicrob doi: 10.1186/1476-0711-11-34.

8. Burkhart PV, Sabaté E (2003) Adherence to long-term therapies: Evidenced for Action. J Nurs Scholarsh 35: 207.

9. USAID (2011) How to Investigate Adherence to Antiretroviral Therapy. An Indicator- Based Approach. USA.

10. Reisner SL, Mimiaga MJ, Skeer M, Perkovich B, Johnson CV, et al. (2009) A review of HIV anti-retroviral adherence and intervention Studies among HIV infected youth. Top HIV Med 17: 14-25.

11. UNAIDS (2007) Greater involvement of people living with HIV (GIPA).

12. Kagee A (2004) Treatment adherence in Ethiopia primary health care: Ethiopia family Practice. SA Fam Pract 46: 26-30.

13. Louie J, Markowit Z (2010) Adherence assessment to highly active Antiretroviral therapy: AIDS patient care, US.

14. Creswell J, Hanson WE, Clark Plano VL, Morales A (2007) Qualitative Research Designs: Selection and Implementation. University of Nebraska Lincoln, SAGE Published, London.

15. Durrhin A, Painter Y (2006) Hand book of qualitative research, sage: London.

16. Mugenda O, Mugenda AG (2003) Research methods: Qualitative \& quantitative approach, acts press: Nairobi.

17. Krueger R (1988) Focus Groups. Thousand Oaks CA: Sage Publication.

18. Yolandi S (2003) Primary caregivers' experiences of rising children with autism: A phenomenological perspective. MA thesis of Rhodes University, Grahamstown.

19. Hardy H, Kumar V, Doros G, Farmer E, Drainoni ML, et al. (2008) Randomized Controlled Trial of a Personalized Cellular Phone Reminder System to Enhance Adherence to Antiretroviral Therapy. AIDS Patient Care STDS 25: 153-61.

20. UNAIDS (2005) AIDS epidemic update December 2005. 


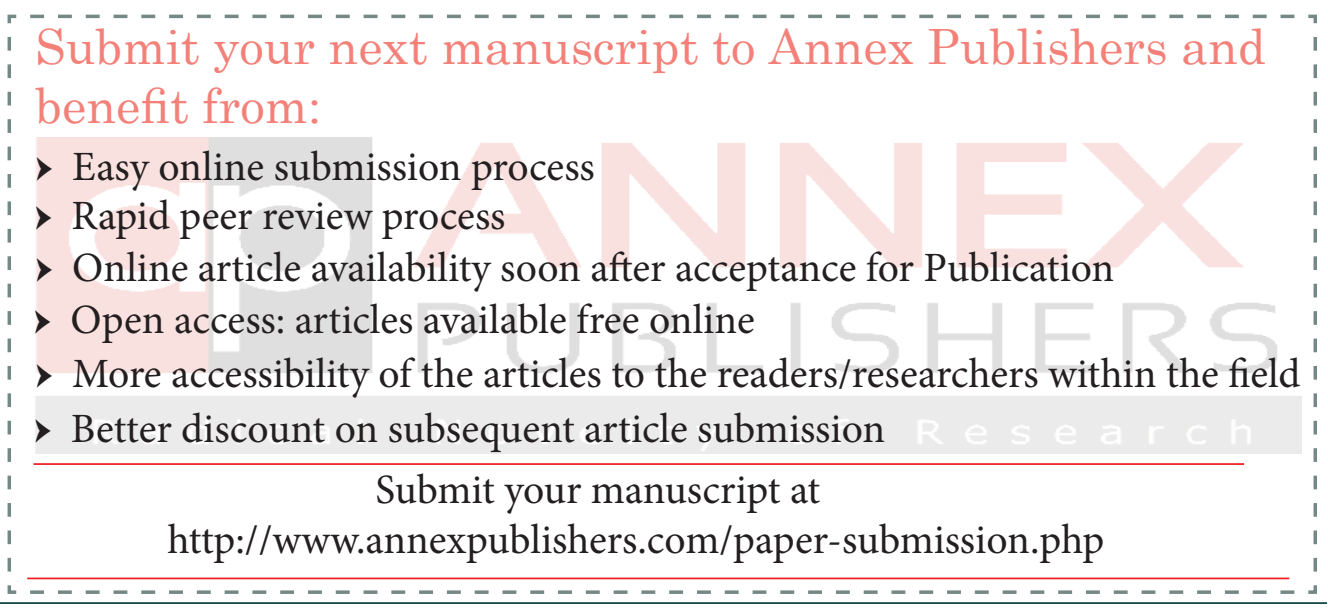

